JOURNAL CLUB

\title{
Improving antibiotic prescribing in acute respiratory tract infections
}

This Norwegian cluster randomised controlled trial assessed the effects of multifaceted educational interventions on antibiotic prescribing for acute respiratory tract infections (RTI) in general practice. Authors of the study hoped to reduce prescription rates and use of broad spectrum antibiotics with their intervention. Seventy-nine medical education groups, totalling 382 general practitioners (GPs), were recruited in the study.

The intervention consisted of two visits by specially trained GPs; one detailing national guidelines for antibiotic use and recent research on acute RTI, and the second to issue reports on individual antibiotic prescribing rates from the preceding year. Primary outcome measures were prescribing rates, and proportion of non-penicillin $\mathrm{V}$ (N-PV) prescription rates before and after intervention. The control arm received a different educational intervention regarding more appropriate prescribing in patients $<70$ years of age.

An adjusted, multilevel model showed a reduction in prescribing rates of antibiotics for acute RTI (OR 0.72 ; $95 \%$ CI 0.61 to 0.84 ) compared with controls. Similarly, there was a reduction in the odds of prescribing a N-PV (OR 0.64; 95\% CI 0.49 to 0.92 ). There was no indication of a stronger intervention effect on GPs with higher baseline prescription rates.

The findings of this study are limited by the fact that educational interventions were delivered by 13 different specially trained GPs. Individual personalities may influence the effect of intervention. Furthermore, especially relevant for the acute RTI group, the data did not distinguish between primary contacts and follow-ups from hospital discharges; therefore prescription data in primary care may underestimate antibiotic rates for this specific group.

- Gjelstad S, Hoye S, Straand J, et al. Improving antibiotic prescribing in acute respiratory tract infections: cluster randomised controlled trial from Norwegian general practice (prescription peer academic detailing (Rx-PAD) study). BMJ 2013;347:f4403.

Leanne Eddie

Correspondence to Dr Leanne Eddie, Poole Hospital NHS Foundation Trust, Longfleet Road, Poole BH15 2JB, UK; leanne.eddie@poole.nhs.uk

To cite Eddie L. Thorax 2014;69:996.

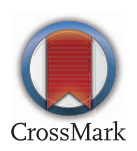

Published Online First 5 November 2013

Thorax 2014;69:996. doi:10.1136/thoraxjnl-2013-204662 